\title{
Versatile genetics
}

\author{
We are constantly amazed by the power of genetics and its ability to solve complex and seemingly intractable \\ problems. The creative application of genetic and genomic analyses to diverse areas has led to advances across \\ basic biology and human disease. We hope to continue to see technologies develop that expand the genetic and \\ genomic toolkit and that bring new discoveries and insights into basic and applied research.
}

T he genetics and genomics fields are growing so rapidly, and expanding into so many sectors, that sometimes it is good to take stock and appreciate the clever applications of genetics and genomics techniques or principles to research questions that remain challenging to address. This month, we highlight a trio of studies that utilize genetics in large-scale and innovative ways.

One of the greatest promises of genomewide association studies (GWAS) is their potential to prioritize genes and pathways as targets for drug therapy. This geneticsled approach to drug-target discovery has been challenging to implement, but advances in analyses of large genomic datasets across different diseases are bringing genetically driven drug prioritization closer to clinical reality. As a robust demonstration of such an advance, Fang et al. (see also accompanying News \& Views) have developed a genetically informed drug-target-prioritization pipeline (termed 'priority index', or Pi). This framework integrates GWAS variants with genomic information to construct a gene-predictor matrix used to prioritize approximately 15,000 genes for 30 immunological traits. The authors showed that incorporating network connectivity information increases enrichment for established therapeutic targets. Importantly, they observed no enrichment for known immunological drug targets when nonimmune-disease GWAS data were used as input. The authors were able to perform cross-trait analysis and found that phenotypically related diseases had similar drug profiles. The Pi system is openly available to the community and can be used to facilitate drug-target prioritization. This approach provides a strong example of how GWAS data can be harnessed to increase the translatability of genetic findings.

Another interesting use of genetics is demonstrated by two studies in this issue, which have used epistatic-interaction information to determine 3D protein structure. Schmiedel and Lehner and Rollins et al. (see also accompanying News \& Views) followed similar strategies for structural determination, by using activity measurements of mutant variants obtained from deep mutational scanning. They found that inter- and intramolecular contacts can be predicted from pairwise epistasis data, and they demonstrated that the sequence positions with the strongest positive epistasis have the closest proximity in the $3 \mathrm{D}$ protein structure. Using a combination of mutagenesis, epistasis analysis and computational tools, the authors took welldescribed genetic concepts and applied them to protein-structure determination, thus highlighting the range of disciplines that genetic analysis can inform.

Reporter assays are of course a staple of genetic analysis, but van Arensbergen et al. present an analysis of human genetic variants on an unprecedentedly large scale (a 100-fold increase over previous studies), using a massively parallel reporter assay for regulatory function. They analyzed
5.9 million SNPs from four genomes in two different cell lines by using their survey of regulatory elements (SuRE) technology, a plasmid-based reporter that can identify both promoter and enhancer activity. This system can test alleles in different local sequence contexts and provide a robust statistical framework for evaluating biological function. The authors identified almost 20,000 reporter assay quantitative trait loci and analyzed overlapping transcription-factor-binding motifs. Although there are some limitations inherent to plasmid-based reporter assays, this large-scale approach allows for the generation of multiple constructs that span the same variant. As such, the analysis is well powered and statistically robust, representing the adaptability and scalability of standard genetics techniques applied in the big-data era.

These examples of using genetic data to prioritize drug targets for disease treatment, combining mutagenesis and epistasis analysis to determine $3 \mathrm{D}$ protein structure, and extending plasmid-based reporter assays to large depth and scale, showcase only a small fraction of the possibilities of how genetics approaches can be applied to various questions in biology. We look forward to seeing continued creative uses of genetics implemented in new, synergistic or scaled-up ways.

Published online: 28 June 2019 https://doi.org/10.1038/s41588-019-0467-y 